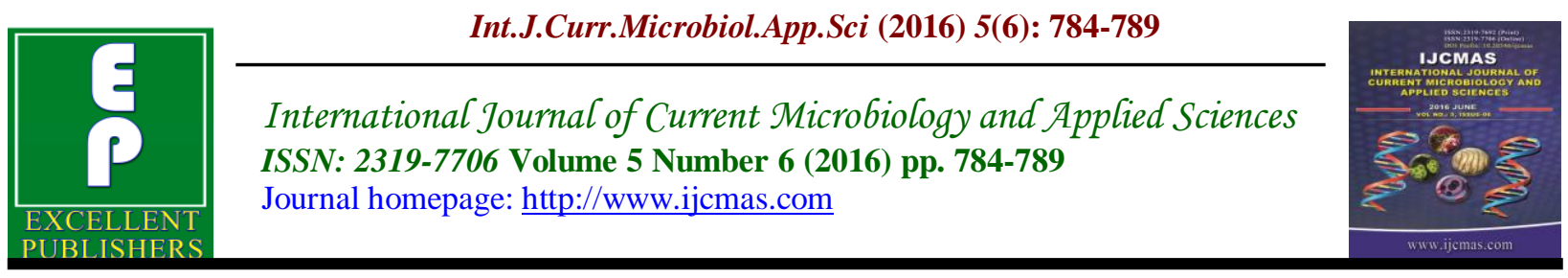

Original Research Article

http://dx.doi.org/10.20546/ijcmas.2016.506.087

\title{
Study of Uropathogenic Multidrug Resistant Escherichia coli and Klebsiella Species with Reference to Extended Spectrum Beta Lactamase and Amp C Beta Lactamase Detection by Phenotypic Method
}

Surender Kaur* and Abhijit A wari
Department of Microbiology, Peoples College of Medical Sciences and Research Centre,
Bhopal-462037 (M.P), India
*Corresponding author email id:

\section{Introduction}

Urinary tract infection is one of the most common bacterial infections in humans, both in the community as well as in the hospital settings. The production of $\beta$ lactamases is the foremost mechanism of antibiotic resistance leading to treatment failure. The $\beta$-lactamases which confer resistance to extended-spectrum cephalosporins are extended-spectrum $\beta$ lactamases (ESBLs) and Amp C. Extended- spectrum beta-lactamases (ESBLs) were first reported in 1983, and plasmid-mediated AmpC beta- lactamases were reported in 1988. ESBLs are Ambler class A or D $\beta$ lactamases which confer resistance to $3 \mathrm{rd}$ and 4th generation cephalosporins and monobactams but are inhibited by cephamycins and $\beta$-lactamase inhibitors like clavulanic acid (CA), sulbactam, and tazobactam. These enzymes are most 
commonly produced by Klebsiella spp. and Escherichia coli but may also occur in other gram-negative bacteria.

AmpC are class $\mathrm{C} \beta$-lactamases which confer resistance to a variety of $\beta$-lactams, including oxyimino-cephalosporins and some cephamycins as well as penicillins and monobactam, when they are produced in large amounts but they are poorly inhibited by $\beta$-lactamase inhibitors such as $\mathrm{CA}$ and sulbactam.

This study shows the changing antibiotic resistance pattern is pertinent for an appropriate treatment and for the prevention and control of the different mechanisms of resistance. So, considering in view of all these facts, study was planned with the following objectives

To find out the drug option for the treatment of UTI due to Escherichia coli and Klebsiella species in the current scenario of increasing antimicrobial resistance, with special reference to ESBL and AmpC $\beta$ lactamase.

\section{Materials and Methods}

Bacterial isolates: A total of 115 consecutive, non-repetitive, clinical isolates which were obtained from the patients of UTI in the clinical bacteriology laboratory, Peoples College of Medical Sciences and Research Centre, Bhopal, (M.P) from October 2012 to September 2013, were included in the study. Urine samples were cultured using $.001 \mathrm{ml}$ loop on CLED, incubated at $37^{\circ} \mathrm{C}$ for $18-24 \mathrm{hrs}$ and number of colonies was counted. Significant bacteuria was defined as greater than $10^{5}$ $\mathrm{CFU} / \mathrm{ml}$ of single pathogen. Isolates were identified by standard procedure.

Antimicrobial susceptibility testing: The isolates were tested by Kirby-Bauer disc diffusion method on Muller Hinton agar (HiMedia), by following the zone size criteria as per CLSI guidelines. The antibiotics $(\mu \mathrm{g})$ which were included for the gram negative isolates were amikacin (30), piperacillin (100), piperacillin/tazobactum (100/10), cefepime (30), cefotaxime (30), ceftriaxone (30), ceftazidime (30), amoxyclav (20/10), cotrimoxazole (25), norfloxacin (10), imipenam (10), nitrofurantoin (300) and cefoxitin (30).

Criteria for the selection of the ESBL producing strains: These isolates were tested for their susceptibility to the third generation cephalosporins (3GCs) e.g. ceftazidime (30 $\mu \mathrm{g})$, cefotaxime $(30 \mu \mathrm{g})$ and ceftriaxone $(30$ $\mu \mathrm{g})$ by using the standard disc diffusion method, as per CLSI guidelines. Zone diameter of $<22 \mathrm{~mm}$ for Ceftazidime (CAZ) was considered to be "suspicious" for ESBL production.

\section{The Phenotypic Confirmatory Disc Diffusion Test (PCT)}

All the strains which were screened out for the ESBL production were subjected to confirmation by using the PCT, as per recommended by the CLSI. In this test, ceftazidime $(30 \mu \mathrm{g})$ discs alone and in combination with clavulanic acid (Ceftazidime + clavulanic Acid, 30/10 $\mu \mathrm{g}$ ) discs, placed on Mueller Hinton Agar (MHA) which was inoculated with the test strain. An increase of $\geq 5 \mathrm{~mm}$ in the zone of inhibition of the combination discs in comparison to that of the Ceftazidime disc alone was considered to be a marker for ESBL production.

Criteria for the selection of the Amp c producing strains: Screening of clinical isolates of E. coli and Klebsiella was performed with Cefoxitin disc $(\mathrm{CX})$. The 
isolates that yielded a CX zone diameter $<18$ mm was CX screen positive. Both screen positive and negative isolates were subjected for confirmation of AmpC enzyme by phenotypic test, Modified Hodge Test.

\section{Modified Hodge test}

All the strains which were screened out for the ESBL production were subjected to Amp c detection by Modified Hodge test (MHT). Lawn culture of E.coli Atcc strain 25922 was prepared, Cefoxitin disc $(30 \mu \mathrm{g})$ was placed at centre and test stain was streaked towards the streak. If the test organism showed Amp c, it hydrolyzed Cefoxitin and showed growth along intersection of the streak and the zone of inhibition from Cefoxitin disk.

\section{Result and Discussion}

The present study was conducted in clinical bacteriology laboratory, Peoples College of Medical Sciences and Research Centre, Bhopal, (M.P) from October 2012 to September 2013 to know antibiotic resistance pattern in uropathogen E.coli and Klebsiella spp.

Antibiotic sensitivity (Table-1) observed in isolates was highest sensitive for Imipenem (96.5\%) followed by Cefoxitin (88.6\%), Piperacillin Tazobactum (80.8\%).

Out of total 115 isolates, 52 were suspicious ESBl (Table-2) which were processed for confirmation of ESBl and AmpC production by PCT and MHT respectively, found to be $53.8 \%$ ESBL and 25\% Amp C producers (Table-3). ESBL producers were maximum resistant for Piperacillin (82.1\%), Cotrimoxazole $(75 \%)$ and Norfloxacin (71.2\%), hence we were left over with parentral drugs only (Table-4).
AmpC producers showed maximum resistant for Piperacillin (92.3\%), Cefepime $(84.6 \%)$ and Cotrimoxazole $(84.6 \%)$ (Table$5)$. Henceforth AmpC producers were more resistant to cephalosporin than Non AmpC producers.

The study revealed E. coli and Klebsiella species to be the dominant organisms among other uropathogens, which coincides with study by Moyo et al., 2010.

As antimicrobial susceptibility patterns vary with region. Isolates in current study showed maximum resistance for Cotrimoxazole followed by Amoxicillin clavulanate, and Norfloxacillin. This may be due to wide use of these drugs empirically as are cheap and oral antibiotic. In the outpatient setting, oral antibiotics are preferred for administration but here we are left with very limited options of oral drugs for the treatment of UTI.

Current study showed percentage of ESBL as $53.8 \%$, AmpC 25\% and ESBL and AmpC coproducers $5.7 \%$ in urinary pathogen which was less than study by Dalela et al., 2012.

Co-existence of both AmpC $\beta$-lactamase and ESBL has been detected in many studies this could be because plasmid mediated AmpC $\beta$-lactamase which has been disseminated among the Enterobacteriaceae. It has seen that AmpC $\beta$-lactamase when present along with ESBLs can mask phenotypic detection of latter. These strains may not be detected by phenotypic method. It may be one of the limitations of the study, which could not detect 08 isolates.

Thus there is need of reliable phenotypic test to identify AmpC $\beta$-lactamase and to discriminate AmpC and ESBL co producers. 
Table.1 Antibiotic susceptibility pattern of E.coli and Klebsiella spp. isolates (n=115)

\begin{tabular}{|l|c|c|c|}
\hline Antibiotics & Sensitive (\%) & Intermediate (\%) & Resistant (\%) \\
\hline Amikacin & $87(75.6)$ & $03(2.6)$ & $25(21.7)$ \\
\hline Norfloxacin & $32(27.8)$ & $02(1.7)$ & $81(70.4)$ \\
\hline Cefepime & $93(80.8)$ & 00 & $22(20.8)$ \\
\hline Piperacillin & $28(24.3)$ & $01(0.8)$ & $86(74.7)$ \\
\hline Piperacillin/tazobactam & $93(80.8)$ & 00 & $22(20.8)$ \\
\hline Imipenam & $111(96.5)$ & 00 & $04(03.4)$ \\
\hline Co-trimoxazole & $21(18.2)$ & $03(2.6)$ & $91(79.1)$ \\
\hline Amoxycillin/clavulanate & $23(20.0)$ & $02(1.7)$ & $90(78.2)$ \\
\hline Nitrofurantoin & $61(53.0)$ & $05(4.3)$ & $49(42.6)$ \\
\hline Cefoxitin & $102(88.6)$ & 00 & $13(11.3)$ \\
\hline Cefotaxime & $70(60.8)$ & 00 & $45(39.1)$ \\
\hline Ceftriaxone & $69(60.0)$ & $01(0.8)$ & $45(39.1)$ \\
\hline Ceftazidime & $63(54.7)$ & 00 & $52(45.2)$ \\
\hline
\end{tabular}

Table. 2 Screening of ESBL, AmpC $\beta$-lactamase among E.coli and Klebsiella spp.

\begin{tabular}{|l|l|l|}
\hline Isolates & $\begin{array}{l}\text { Screening positive ESBL } \\
\text { CAZ } \leq 22 \mathrm{~mm}\end{array}$ & $\begin{array}{l}\text { Screening positive AmpC } \\
\mathrm{CX}<18 \mathrm{~mm}\end{array}$ \\
\hline E.coli & $33(63.4 \%)$ & $11(84.6 \%)$ \\
\hline Klebsiella spp & $19(36.5 \%)$ & $02(15.3 \%)$ \\
\hline Total & $52(45.21 \%)$ & $13(11.3 \%)$ \\
\hline
\end{tabular}

Table.3 Prevalence of ESBL and AmpC $\beta$-lactamase among E.coli and Klebsiella spp

\begin{tabular}{|l|l|l|l|l|}
\hline Isolates & ESBL & Amp C & $\begin{array}{l}\text { ESBL and AmpC co } \\
\text { producers }\end{array}$ & Not detected \\
\hline E.coli & $13(46 \%)$ & $11(84 \%)$ & 02 & 07 \\
\hline Klebsiella spp & $15(53 \%)$ & $02(15.3 \%)$ & 01 & 01 \\
\hline Total & $28(53.8 \%)$ & $13(25 \%)$ & $03(5.7 \%)$ & $08(21.1 \%)$ \\
\hline
\end{tabular}

Table.4 Comparison of antibiotic resistance pattern between ESBL and non ESBL producers

\begin{tabular}{|l|l|l|}
\hline Antibiotics & ESBL $(\mathbf{n = 2 8 ) \%}$ & Non ESBL (n= 87)\% \\
\hline Amikacin & $08(28.5)$ & $17(19.5)$ \\
\hline Norfloxacin & $20(71.2)$ & $61(70.1)$ \\
\hline Cefepime & $19(67.8)$ & $05(05.7)$ \\
\hline Piperacillin & $23(82.1)$ & $63(72.4)$ \\
\hline Piperacillin/tazobactam & $05(17.8)$ & $19(21.8)$ \\
\hline Imipenam & $01(3.5)$ & $03(03.4)$ \\
\hline Co-trimoxazole & $21(75.0)$ & $70(80.4)$ \\
\hline Amoxycillin/clavulanate & $17(60.7)$ & $73(83.9)$ \\
\hline Nitrofurantoin & $14(50.0)$ & $35(40.2)$ \\
\hline Cefoxitin & $00(00)$ & $13(14.9)$ \\
\hline Cefotaxime & $23(82.1)$ & $22(25.2)$ \\
\hline Ceftriaxone & $22(78.5)$ & $23(26.4)$ \\
\hline Ceftazidime & $28(100)$ & $24(27.5)$ \\
\hline
\end{tabular}


Table.5 Comparison of antibiotic resistance pattern between AmpC and non AmpC producers

\begin{tabular}{|l|l|l|}
\hline Antibiotics & Amp C $(\mathbf{n = 1 3})$ & Non Amp C (n=102 ) \\
\hline Amikacin & $10(76.9)$ & $15(14.7)$ \\
\hline Norfloxacin & $11(84.6)$ & $70(68.6)$ \\
\hline Cefepime & $11(84.6)$ & $13(12.7)$ \\
\hline Piperacillin & $12(92.3)$ & $74(72.5)$ \\
\hline Piperacillin/tazobactam & $09(69.2)$ & $15(14.7)$ \\
\hline Imipenam & $00(00.0)$ & $04(3.9)$ \\
\hline Co-trimoxazole & $11(84.6)$ & $80(78.4)$ \\
\hline Amoxycillin/clavulanate & $13(100)$ & $77(75.4)$ \\
\hline Nitrofurantoin & $09(69.2)$ & $40(39.2)$ \\
\hline Cefoxitin & $13(100)$ & $00(00.0)$ \\
\hline Cefotaxime & $07(53.8)$ & $38(37.2)$ \\
\hline Ceftriaxone & $06(46.6)$ & $39(38.2)$ \\
\hline Ceftazidime & $09(69.2)$ & $43(42.1)$ \\
\hline
\end{tabular}

Our study also corroborates the finding that ESBL and Amp $\mathrm{C}$ producing isolates are much more multidrug-resistant than ESBLsnegative and Amp C-negative isolates thereby, narrowing down the choice of antibiotics for treatment. Considering above findings, there is a dire need of introducing some new antimicrobial drug for UTIs.

In conclusion, multidrug resistant strains of E.coli and Klebsiella spp. are widely prevalent and their isolation in UTI is a matter of grave concern. Our study shows a high degree of MDR E. coli and Klebsiella spp. which showed resistance to five and six groups of antibiotics and nearly $50 \%$ exhibited ESBL production and 25\% Amp C production.

Most of these isolates including the ESBL and Amp C strains were sensitive to Imipenem, Piperacillin-tazobactum and Amikacin. Antibiotics like Imipenem being a parentral drug require hospitalization and drug monitoring, all of which incurs high cost to the patient and cannot be used as the first line of treatment. Inspite of low percentage of resistance to Imipenem in our study, the threat of spread of carbapenemases still looms large. Hence, the use of carbapenems has to be restricted to complicated and long standing UTIs. Judicious use of antibiotics is the need of the hour to prevent spread of the multidrug resistant strains in the community.

\section{References}

Bauernfeind, A., Chong, Y., Lee, K. 1998. Plasmid-encoded AmpC betalactamases: how far have we gone 10 years after the discovery? Yonsei Med. J., 39: 520-5.

Bauernfeind, A., Chong, Y., Schweighart, S. 1989. Extended broad- spectrum Blactamase in Klebsiella pneumoniae including resistance to cephamycins. Infect., 17: 316-21.

Deepika Handa, Anita Pandey. 2013. Evaluation of phenotypic tests for the detection of AmpC beta-lactamase in clinical isolates of Escherichia coli. Indian J. Pathol. Microbiol., 56(2): 135-138.

Gaurav Dalela, SwetaGupta, Dinesh Kumar Jain, PushpaMehta. 2012. Antibiotic resistance pattern in uropathogens at tertiary care hospital at Jhalwar with special reference to ESBl, Amp c $\beta$ lactamase and MRSA production $J$. 
Clin. Diag. Res., (Suppl-2), Vol-6(4): 645-651.

Goussard, S., Courvalin, P. 1999. Updated sequence information for TEM betalactamase genes. Antimicrob Agents Chemother ., 43: 367-70.

Kenneth, H. 2011. etalClinical Laboratory Detection of AmpC $\boldsymbol{\beta}$-Lactamase Does It Affect Patient Outcome? Am. J. Clin. Pathol., 135: 572-576.

Koneman, E.W., Allen, S.D., Janda, W.M., Schreckenberger, P.C. The Enterobacteriaceae. In:Koneman's color atlas and textbook of diagnostic microbiology, 6th ed (pp 211-302). Philadelphia, PA: Lippincott Williams \& Wilkins, 2006a.

Kothari, A., Sagar, V. 2008. Antibiotic resistance in pathogens causing community-acquired urinary tract infections in India: A multicenter study. J. Infect. Dev. Ctries, 2: 354-8.

Payne, D.J., Cramp, R., Winstanley, D.J., Knowles, D.J. 1994. Comparative activities of clavulanic acid, sulbactam, and tazobactam against clinically important beta-lactamases. Antimicrob Agents Chemother., 38: 767-72.
Ratna, A.K,. Menon, I., Kapur, I., Kulkarni, R. 2003. Occurrence \& detection of AmpC beta-lactamases at a referral hospital in Karnataka. Indian J. Med. Res., 18: 29-32.

Sasirekha, B. 2013. prevalence of ESBL, Amp C $\beta$-lactamases and MRSA among uropathogen and its Antibiogram. EXCLI J., 12: 81-88 ISSN 1611-2156.

Smitha, O., Bagali, B.V. Peerapur. 2013. Detection of AmpC Beta-lactamases among Escherichia coli isolates at a tertiary care hospital in Karnataka. $J$. Med. Sci., 6(1): 85-87

Yagi, T., Wachino, J., Kurokawa, H., Suzuki, S., Yamane, K., Doi, Y., et al. 2005. Practical methods using boronic acid compounds for identification of class $\mathrm{C}$ beta-lactamase producing Klebsiella pneumoniae and Escherichia coli. J. Clin. Microbiol., 43: 2551-8.

Yilmaz, N.O., N. Agus, E. Bozcal, O. Oner, A. Uzel. 2013. Detection of plasmidmediated AmpC $\beta$-lactamase in Escherichia coli and Klebsiella Pneumoniae. Indian J. Med. Microbiol., 31(1): 53-59.

\section{How to cite this article:}

Surender Kaur and Abhijit Awari 2016. Study of Uropathogenic Multidrug Resistant Escherichia coli and Klebsiella Species with Reference to Extended Spectrum Beta Lactamase and Amp C Beta Lactamase Detection by Phenotypic Method. Int.J.Curr.Microbiol.App.Sci. 5(6): 784-789. doi: http://dx.doi.org/10.20546/ijcmas.2016.506.087 